\title{
Paniculitis eosinofílica autoinducida: un desafío diagnóstico
}

\author{
N. GÓMEZ RODRÍGUEZ*, J. A. ORTIZ-REY, A. DE LA FUENTE BUCETA, \\ J. IBÁÑEZ RUÁN* \\ *Unidad de Reumatología y Servicio de Anatomía Patológica. Centro Médico POVISA. \\ Vigo
}

EOSINOPHILIC PANNICULITIS AUTOINDUCED

\begin{abstract}
RESUMEN
Las paniculitis eosinofílicas se caracterizan por una intensa infiltración de la hipodermis por eosinófilos y, aunque la morfología clínica de las lesiones es variada, predominan las formas nodulares. Su amplio espectro etiológico está dominado por causas sistémicas. Las paniculitis eosinofilicas facticias son excepcionales. Como otras paniculitis autoinducidas, tienen un gran pleomorfismo clínico y se asocian con trastornos de la personalidad. El principal problema que plantean es su correcta filiación diagnóstica. Presentamos el caso de una paniculitis nodular febril localizada en antebrazo y brazo izquierdos de una paciente de 16 anos, autoinducida mediante la inyección de preparados agrícolas para fumigación, en el contexto de un intento de autolisis. Comentamos el cuadro clínico, los hallazgos radiográficos y su diagnóstico diferencial.
\end{abstract}

PALABRAS CLAVE: Paniculitis facticia. Intento de autolisis. Paniculitis nodular autoinducida. Paniculitis eosinofílica.

\begin{abstract}
Eosinophilic panniculitis is characterized by a prominent infiltration of subcutaneous fat with eosinophils. The clinical spectrum of lesions that display pathologic changes of eosinophilic panniculitis is diverse, but nodular lesions are the most common. The factitial eosinophilic pan niculitis are exceptional, show a pleomorphic pattern of findings and are associated with personality disorders. Its main challenge is to establish the correct diagnostic. We report an unusual case of factitial nodular febrile panniculitis of the left forearm and proximal arm in an 16yearold girld. She admitted that the lesions were self-inflicted by autoinjec tion of several farming products as attempted suicide. We describe the clinical, radiological and histopathological features as well as the diffe rential diagnosis of this type of panniculitis.
\end{abstract}

KEY WORDS: Factitial Panniculitis. Self-inflicted nodular panniculitis. Attempted suicide. Eosinophilic panniculitis.

Gómez Rodríguez N, Ortiz-Rey JA, De la Fuente Buceta A, Ibáñez Ruán J. Paniculitis eosinofílica autoinducida: un desafío diagnóstico. An Med Interna (Madrid) 2001; 18: 635-637.

\section{INTRODUCCIÓN}

Las paniculitis incluyen un amplio grupo de procesos en los que el foco inflamatorio, generalmente múltiple, se localiza en la hipodermis. Dentro de su amplio espectro etiológico, las formas autoinducidas son especialmente difíciles de diagnosticar, ya que rara vez se piensa en ellas y los enfermos suelen acudir al médico general o al dermatólogo, eludiendo la atención psiquiátrica (1). Excepcionalmente, se han señalado casos producidos por técnicas de medicina alternativa como la acupuntura o las ventosas (2). Las sustancias utilizadas para ello son de lo más diverso (3), pero los fungicidas de uso agrícola, en nuestro conocimiento, constituyen una novedad, hecho que asociado a la escasez de comunicaciones sobre paniculitis facticias en la literatura nacional, nos ha llevado a aportar este nuevo caso.

\section{CASO APORTADO}

Una adolescente de 16 años, procedente del medio rural, consultó por dolor y tumefacción en el miembro superior izquierdo, a ambos lados de la flexura del codo. Refería que los síntomas se iniciaron de forma aguda, se asociaron a fiebre termometrada $\left(38,5-39^{\circ}\right.$ C), y fueron precedidos por la picadura de un artrópodo que no llegó a identificar. Negaba hábitos tóxicos o traumatismos locales. Se procedió a su ingreso y, en la exploración, se apreciaron tres nódulos eritematosos con distribución lineal, uno en el tercio superior de la cara volar del antebrazo izquierdo, y dos en el borde cubital del brazo, inmediatamente por encima del codo, con bordes mal delimitados y consistencia dura. Su diámetro era, respectivamente de 4,1, 3,5 y 3 $\mathrm{cm}$. La movilidad del codo estaba secundariamente restringida, tanto activa como pasivamente (recorrido de flexoextensión: $15^{\circ}-80^{\circ}$ ), pero sin derrame o sinovitis. Se palpaban adenopatías axilares izquierdas indoloras, de consistencia elástica, así como una adenopatía epitroclear. No se identificaron posibles puertas de entrada de

Trabajo aceptado: 19 de Mayo de 2000

Correspondencia: Norberto Gómez Rodríguez. Unidad de Reumatología. Centro Médico POVISA. C/ Salamanca n5, 362100 Vigo. Fax: 98642 14 39 
microorganismos. La enferma se encontraba febril $\left(39,5^{\circ} \mathrm{C}\right)$ pero mantenía un buen estado general. No se palpaban visceromegalias ni masas abdominales y tanto la auscultación cardiopulmonar como el examen neurológico fueron normales.

El hemograma mostró: leucocitos $(12,6$ x 10\%/L) con $81 \%$ de neutrófilos, $11 \%$ linfocitos, $7 \%$ monocitos y $1 \%$ eosinófilos, $\mathrm{Hb}(130$ $\mathrm{g} / \mathrm{L})$, plaquetas (181 X 10\%/L). VSG: $48 \mathrm{~mm} / \mathrm{l}^{\mathrm{a}} \mathrm{h}$. Bioquímica (glucosa, urea, creatinina, calcio, fósforo, creatincinasa, transaminasas, lácticodehidrogenasa, sodio y potasio), proteinograma, así como la dosificación del complemento sérico (CH50, C3 y C4) fueron normales. Fibrinógeno $(0,517 \mathrm{~g} / \mathrm{L})$. El factor reumatoide (látex) y los anticuerpos antinucleares fueron negativos. Las radiografías simples mostraron aumento del volumen y densidad de partes blandas en el extremo superior del antebrazo izquierdo.

Durante el tercer día de estancia el nódulo del antebrazo se ulceró y comenzó a drenar un exudado parduzco cuyo cultivo permitió aislar enterococos. Tres hemocultivos fueron negativos. La resonancia magnética evidenció alteraciones de la señal en el extremo proximal del antebrazo izquierdo y en su borde cubital, compatibles con celulitis, sin signos de afección articular (Fig. 1). Se inició el tratamiento con ampicilina y gentamicina, que tuvo que interrumpirse al quinto día por la aparición de un síndrome vertiginoso. Aunque la fiebre desapareció, los nódulos permanecieron invariables. La paciente fue dada de alta, pero fue reingresada dos meses después por reaparición de la fiebre (hasta $40^{\circ} \mathrm{C}$ ) e intensificación del dolor local. Los hallazgos en la exploración fueron similares a los reseñados, excepto por la aparición de flebotrombosis superficial, linfangitis en tercio proximal de antebrazo y mitad distal del brazo. Los nódulos habían aumentado su consistencia y existía edema con fóvea en todo el antebrazo izquierdo. Se procedió a la biopsia del nódulo del antebrazo cuyo estudio histológico mostró una paniculitis preferentemente lobulillar, con infiltrado inflamatorio de predominio eosinofilico y extensas áreas de necrosis. No se observaron granulomas ni material birrefringente en el estudio con luz polarizada (Fig. 2).

Ante la localización de los nódulos y sus características histológicas, se interrogó exhaustivamente a la paciente y a sus familiares. Pese al hermetismo y la reticencia inicial, la enferma acabó por reconocer que había intentado inyectarse una mezcla fungicida de uso agrícola en las venas, a su paso sobre el codo, como intento de autolisis, pretendiendo así coaccionar a los padres para que aceptasen a su novio. La resolución de los nódulos se produjo muy lentamente en un período de 6 meses, quedando una induración residual.

\section{DISCUSIÓN}

Las dermatosis autoinducidas son lesiones únicas o múltiples, por lo general superficiales, que se localizan en áreas accesibles para el paciente (1,3-6), quien niega sistemáticamente su autoría $(1,3)$. Dentro de este grupo de procesos, son menos habituales los que involucran la hipodermis -paniculitis- y que pueden provocarse por la inyección de irritantes químicos (3) o mediante procedimientos físicos (7). Se han llegado a utilizar los más variados productos, tales como aceites, mostaza, pintura, povidona yodada, orina, leche o heces (3). Los fungicidas para uso agrícola constituyen, en nuestro conocimiento, una curiosa novedad. La reacción inflamatoria provocada por estas sustancias en el caso presentado fue muy intensa y persistente, con escasa respuesta a los antinflamatorios no esteroideos. Todavía en la actualidad, los mecanismos psicológicos implicados en la autolesión distan de ser comprendidos y los trastornos psiquiátricos asociados con las diferentes formas de dermatosis facticias son dispares (1). El clínico puede quedar desconcertado por las características de este tipo de lesiones, variable según la sustancia empleada, así como por su extraña evolución y distribución, hechos que

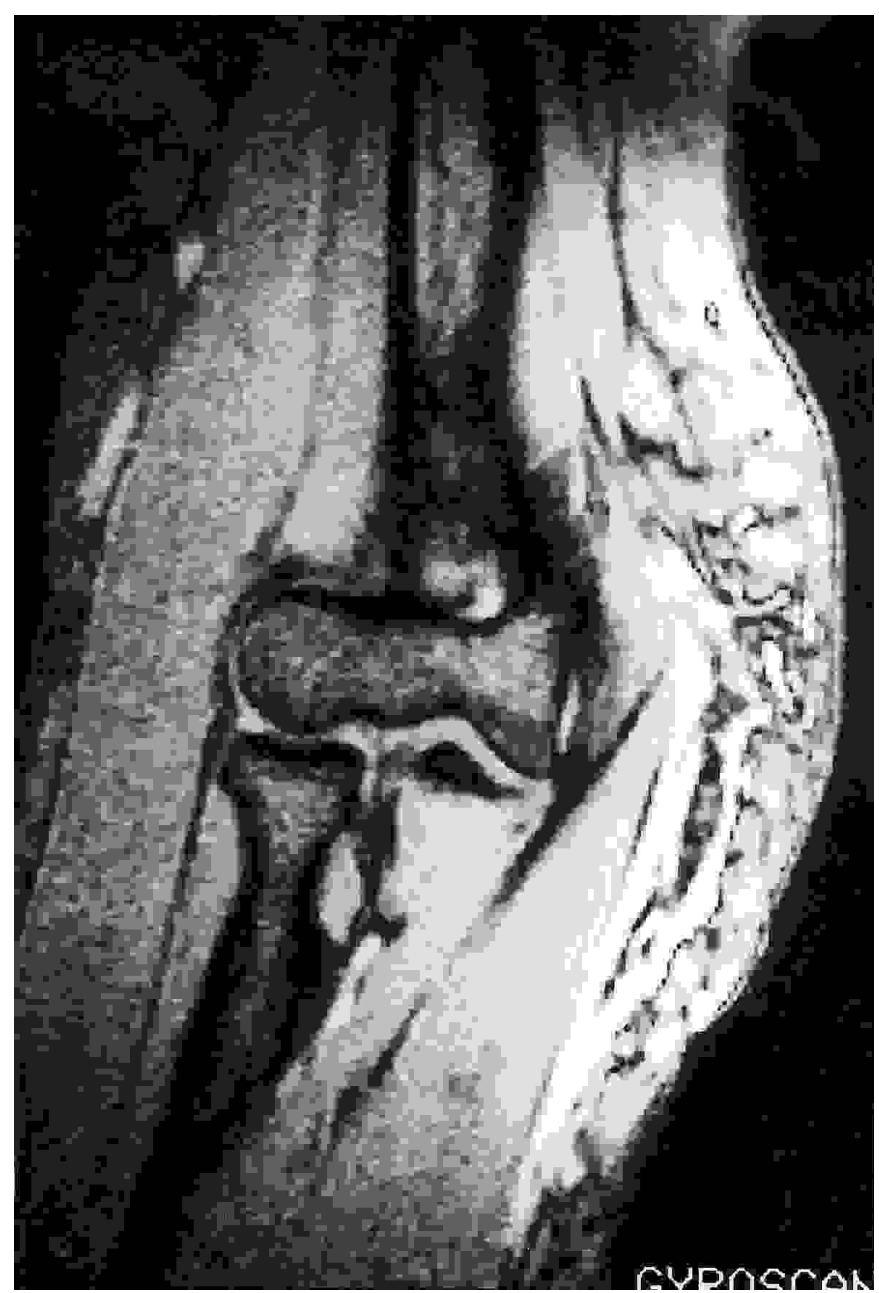

Fig. 1. Corte coronal de reesonancia magnética, ponderado en T2, que involucra el tercio proximal del antebrazo izquierdo. Se observa un aumento de volumen y de a señal en la hipodermis, con escasa afección de los músculos subyacentes y ausencia de alteraciones óseas.

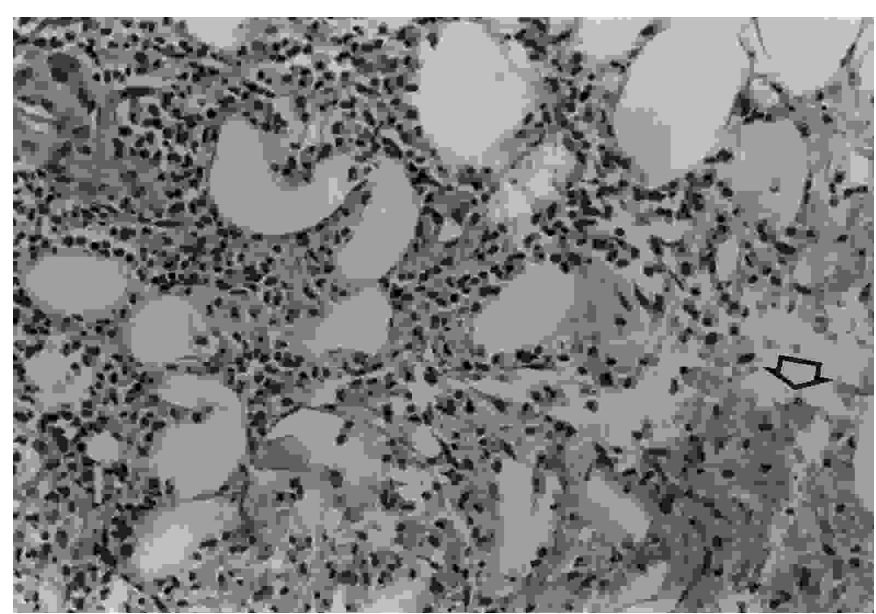

Fig. 2. Imagen de microscopía óptica del tejido hipodérmico afectado: infiltrado inflamatorio lubulillar con predominio de eosinófilos y necrosis $(\leftarrow)$. $(H-E, 200 x)$. 
deben hacer sospechar la posibilidad de que sean autoprovocadas $(1,3)$. Los hallazgos histológicos en las paniculitis facticias son variados e incluyen infiltrados inflamatorios polimorfonucleares, esteatonecrosis, presencia de histiocitos, granulomas, dependiendo, entre otros factores, de la sustancia inyectada (8). El infiltrado eosinofilico, como en el caso aportado, no es tampoco específico y se ha descrito en otras causas de paniculitis como las picaduras de artrópodos, infecciones bacterianas, dermatitis atópica, enfermedades autoinmunes y parasitosis (9). La observación de partículas refráctiles intersticiales al estudiar las muestras con luz polarizada es altamente sugestivo de la inyección hipodérmica de sustancias extrañas $(7,10)$.

\section{Bibliografía}

1. Van Moffaert M. Localization of self-inflicted dermaological lesions: what do they tell the dermatologist? Acta Derm Venereol Suppl (Stockch) 1991; 156: 23-7.

2. Lee JS, Ahn SK, Lee SH. Facticial panniculitis induced by cupping and acupuncture. Cutis 1995; 55: 217-8.

3. Bondi EE, Lazarus GS. Trastornos del tejido subcutáneo. En: Fitzpatrick TB, Eisen AZ, Wolff K, Freedberg IN, Austen KF, eds. Dermatología en Medicina General. $3^{a}$ edición. Buenos Aires, Editorial Médica Panamericana S.A; $1988,1280-97$.

4. Brehmer-Andersson E, Goransson K. Friction blisters as a manifestation of pathomimia. Acta Derm Venereol 1975; 55: 65-71.

5. Sneddon I, Sneddon J. Self-inflicted injury: a follow-up study of 43 patients. Br Med J 1975; 30: 527-30.

6. Urgulu S, Bartley GB, Otley CC, Baratz KH. Factitious disease of periocular and facial skin. Am J Ophthalmol 1999; 127: 196-201.
La adopción de una actitud comprensiva y un abordaje gradual del problema, pueden ayudar al reconocimiento del origen del cuadro por parte del enfermo. Sin embargo, esto no siempre es suficiente para que cesen las autoagresiones (11). Tampoco debe olvidarse que la disposición de las lesiones de algunas dermatosis y su ulterior evolución inducen ocasionalmente la sospecha de autoinducción, por lo que se han llegado a denominar dermatosis pseudofacticias (12). En general, las paniculitis autoinducidas son situaciones comprometidas, verdaderos desafíos diagnósticos para el médico, que requieren de la mayor perspicacia y capacidad de profundización en los aspectos psicológicos del enfermo.
7. Sarkany I, Mcmillan AL. Recurrent nonscarring pressure panniculitis. Proc R Soc Med 1969; 62: 1279.

8. McKee $\mathrm{OH}$. Pathology of the skin with clinical correlations. $2^{\circ}$ Edición. Time Mirror International Publishers Limited; 1996.

9. Adame J, Cohen PR. Eosinophilic panniculitis: Diagnostic considerations and evaluation. J Am Acad Dermatol 1996; 34: 229-34.

10. Antony SJ, Mannion SM. Dermatitis artefacta revisited. Cutis 1995; 55 : 362-4.

11. Wang CK, Lee JY. Monosymptomatic hypochondriacal psychosis complicated by selfinflicted skin ulceration, skull defect and brain abscess. Br J Dermatol 1997; 137: 299-302.

12. Lachapelle JM, Bataille AC, Tennstedt D, Marot L Pseudo-factitial dermatitis: A useful clinical and/or histopathological concept. Dermatology 1994; 189 (suppl 2): 62-4. 